\title{
Short- and long-term predictors of spontaneous bacterial peritonitis in Singapore
}

Yu Jun Wong ${ }^{1}$, MRCP, FAMS, Rajamanickam Chandrasekaran Kalki ${ }^{1}$, MBBS, MRCP, Kenneth Weicong Lin ${ }^{1}$, MBBS, MRCP, Rahul $\underline{K u m a r}^{1}$, MBBS, MRCP, Jessica $\underline{T a n}^{1}$, MBBS, MRCP, Eng Kiong $\underline{T e 0^{1}}$, MBBS, MRCP, James Weiquan $\underline{\operatorname{Li}}^{1}$, MBBS, MRCP, Tiing Leong Ang $^{1}$, FRCPEd, FAMS

INTRODUCTION Spontaneous bacterial peritonitis (SBP) is the commonest complication of liver cirrhosis. Timely and appropriate treatment of SBP is crucial, particularly with the rising worldwide prevalence of multidrug-resistant organisms (MDROs). We aimed to investigate the clinical outcomes of SBP in Singapore.

METHODS All cirrhotic patients with SBP diagnosed between January 2014 and December 2017 were included. Nosocomial SBP (N-SBP) was defined as SBP diagnosed more than 48 hours after hospitalisation. Clinical outcomes were analysed as categorical outcomes using univariate and multivariate analysis.

RESULTS There were 33 patients with 39 episodes of SBP. Their mean age was 64.5 years and $69.7 \%$ were male. The commonest aetiology of cirrhosis was hepatitis B (27.3\%). The Median Model for End-stage Liver Disease (MELD) score was $17 ; 33.3 \%$ had acute-on-chronic liver failure and $60.6 \%$ had septic shock at presentation. N-SBP occurred in $25.6 \%$ of SBP cases. N-SBP was more commonly associated with MDROs, previous antibiotic use in the past three months $(p=0.014)$ and longer length of stay $(p=0.011)$. The 30-day and 90-day mortality among SBP patients was $30.8 \%$ and $51.3 \%$, respectively. MELD score $>20$ was a predictor for 30 -day mortality. N-SBP and MELD score $>20$ were predictors for 90-day mortality.

CONCLUSION N-SBP was significantly associated with recent antibiotic use, longer hospitalisation, more resistant organisms and poorer survival among patients with SBP. N-SBP and MELD score predict higher mortality in SBP. Judicious use of antibiotics may reduce N-SBP and improve survival among cirrhotic patients.

Keywords: nosocomial, predictors, spontaneous bacterial peritonitis, survival

\section{INTRODUCTION}

Spontaneous bacterial peritonitis (SBP) is the commonest complication among patients with liver cirrhosis.(1) SBP can lead to decompensation of liver cirrhosis, acute-on-chronic liver failure (ACLF) with fourfold increased risk of short-term mortality, early re-admission and increased healthcare burden. ${ }^{(2,3)}$ Despite early recognition and treatment according to current guidelines, its 90-day mortality rate remained high, ranging from $43.9 \%$ to $79.4 \% .^{(4-6)}$ This poor outcome is largely attributed to the emergence of multidrug-resistant organisms (MDROs) and Gram-positive organisms in patients with SBP.

The 'leaky gut' theory suggests that SBP is a complication of the translocation of enteric bacteria into ascitic fluid. The three main mechanisms that support this theory among patients with advanced cirrhosis are increased gut permeability, gut dysbiosis and reduced peritoneal immunoglobulin concentration. ${ }^{(7,8)}$ Increased gut permeability occurs as a result of portal hypertension in advance cirrhosis, leading to gut oedema and subsequent loosening of tight junctions, ${ }^{(9-11)}$ particularly among patients with ascites. $^{(12)}$ Gut dysbiosis is well reported among patients with cirrhosis. ${ }^{(13)}$ In particular, cirrhotic patients receiving quinolone prophylaxis have been associated with the emergence of MDROs in SBP. ${ }^{(14)}$ Together with increased gut permeability and gut dysbiosis, patients with advanced cirrhosis were also found to have reduced peritoneal immunoglobulins and impaired opsonisation of translocated enteric organisms, thus leading to an increased risk of SBP in these patients. ${ }^{(15-17)}$

The rising trend of MDROs among patients with SBP is a growing concern. ${ }^{(18)}$ Recognising the global threat of antimicrobial resistance, the World Health Organization (WHO) has endorsed a global action plan to counter antimicrobial resistance and ensure the continuity of effective treatment options for infectious disease. ${ }^{(19)}$ The emergence of MDROs reduces the efficacy of empirical antibiotic treatment for SBP, resulting in prolonged hospitalisation as well as increased healthcare utilisation, morbidity and mortality among patients with SBP. This change in the epidemiology and resistance pattern of the microorganisms causing SBP was attributed to the widespread use of broadspectrum antibiotics, primary prophylaxis for SBP and increasing instrumentation for cirrhosis patients. ${ }^{(20)}$ However, resistance patterns of microorganisms in SBP vary geographically. While the use of broader-spectrum antibiotics for SBP could improve treatment response in a region with a high prevalence of MDROs, the same practice could potentially promote the emergence of MDROs among regions where their prevalence is low. Thus, understanding local resistance patterns is essential in order to tailor our empirical treatment to SBP in Singapore.

Identifying the outcome predictors of SBP is also important, not only in identifying patients who are more likely to benefit from more aggressive empirical treatment, but also to enhance

${ }_{1}^{1}$ Department of Gastroenterology and Hepatology, Changi General Hospital, Singapore

Correspondence: Dr Wong Yu Jun, Associate Consultant, Department of Gastroenterology and Hepatology, Changi General Hospital, 2 Simei Street 3, Singapore 529889. eugene.wong.y.j@singhealth.com.sg 
our understanding of the pathogenesis of SBP. Reported predictors of poor outcome among SBP include MDROs, septic shock, poor liver reserve and nosocomial SBP (N-SBP)..$^{3,5,21-23)}$ These patients may benefit from broad-spectrum antibiotics as an empirical treatment for SBP.

To date, N-SBP remains an under-recognised entity in Singapore. The primary aim of this study was to describe the clinical outcomes of SBP in Singapore, while the secondary aim was to determine the prevalence of N-SBP in Singapore.

\section{METHODS}

This study was conducted at Changi General Hospital, a 1,000bed teaching hospital serving a population of 1.3 million from the east and northeast regions of Singapore. We conducted a retrospective observational study of all SBP diagnosed among patients with liver cirrhosis between January 2014 and December 2017.

Patients were identified with electronic medical records using the procedure code for paracentesis and ICD-10 (International Classification of Diseases, 10th revision) code for liver cirrhosis. All adult patients (age $\geq 18$ years) with a diagnosis of liver cirrhosis and SBP who underwent paracentesis were consecutively included. Data on all patients' baseline characteristics and outcomes were reviewed by the investigator using electronic medical records. Patients were excluded if there was secondary peritonitis, malignant ascites or lack of data on primary outcomes. All patients were followed up for a minimum of six months from the date of hospitalisation. The study protocol was approved by our institutional review board (IRB ref No. 2018/2892).

Paracentesis was performed for all cirrhotic patients who were hospitalised for decompensation with clinical suspicion of SBP and refractory ascites. All paracenteses were performed using sterile technique. The ascitic fluid collected was sent for fluid analysis, which included polymorphonuclear leucocytes (PMN) counting. An additional $10 \mathrm{~mL}$ of ascitic fluid was collected and inoculated into aerobic and anaerobic blood culture bottles for seven days. Positive cultures identified would be tested with the antibiotic susceptibility test using the VITEK® 2 compact system and minimal inhibitory concentration method. An empirical antibiotic was initiated upon the diagnosis of SBP according to international guidelines. (2) Patients with community-acquired SBP (C-SBP) received intravenous ceftriaxone, while patients with $\mathrm{N}$-SBP received intravenous piperacillin/tazobactam. Intravenous albumin was administrated to all patients diagnosed with SBP, as per guideline. ${ }^{(24)}$ We subsequently studied the clinical outcome of SBP, which included 30-day and 90-day mortality, and factors associated with these outcomes.

SBP was defined as PMN $\geq 250$ cells $/ \mathrm{mm}^{3}$ in the ascites fluid or positive culture from the ascites fluid. SBP that was diagnosed more than 48 hours after hospitalisation was defined as N-SBP. SBP that was diagnosed within 48 hours after hospitalisation without recent hospitalisation in the preceding three months was defined as C-SBP. ${ }^{(25)}$ Cirrhosis was defined based on clinical, biochemical, radiological and/or histological findings. The severity of liver cirrhosis was assessed using the Child-Pugh score and the Model for End-stage Liver Disease score with sodium (MELD-Na) and without sodium (MELD) at the point of diagnosis of SBP. The presence of shock upon hospitalisation was defined as systolic blood pressure $<90 \mathrm{mmHg}$ and mean arterial pressure $<65 \mathrm{mmHg}$ in the presence of SBP. Systemic inflammatory response syndrome (SIRS) was defined as fulfilling two or more of the following criteria: fever of $>38^{\circ} \mathrm{C}$ or $<36^{\circ} \mathrm{C}$, heart rate $>$ 90 beats per minute, respiratory rate $>20$ breaths per minute and abnormal white cell count $(>12,000 / \mu \mathrm{L}$ or $<4,000 / \mu \mathrm{L})$. Presence of ACLF was defined based on the CANONIC study definition. ${ }^{(26)}$ Extended-spectrum beta-lactamase-producing (ESBL-producing) organisms were defined as microorganisms with resistance to extended-spectrum (third-generation) cephalosporin and monobactams. MDROs were defined as microorganisms with resistance to at least one agent in three or more antimicrobial categories. $^{(27)}$

All analysis was performed with SPSS Statistics version 21.0 (IBM Corp, Armonk, NY, USA). Continuous variables were computed using Student's $t$-test or Mann-Whitney $U$ test, and categorical variables were computed using chi-square test or Fisher's exact test. Univariate analysis was performed to identify factors associated with study outcomes. All covariates with $p<0.10$ on univariate analysis were retained and included in multivariate analysis. The data was censored at last follow-up, liver transplantation or death. A p-value $<0.05$ was indicative of statistical significance.

\section{RESULTS}

A total of 33 patients with 39 episodes of SBP were studied among 645 patients with cirrhosis. Therefore, the incidence of SBP was $5.1 \%$. Of the patients with SBP, 23 (69.7\%) were male with a mean age of $64.5 \pm 10.9$ years. The commonest aetiology of cirrhosis was chronic hepatitis B (27.3\%) followed by chronic hepatitis C $(24.2 \%)$, non-alcoholic steatohepatitis $(24.2 \%)$ and others (24.2\%). The majority (60.6\%) had a Class B Child-Pugh score, while the remaining patients (39.4\%) were Class C. Median MELD and MELD-Na scores were 17.0 and 18.0, respectively. About $60.6 \%$ of the patients had septic shock and/or SIRS upon presentation, and $33.3 \%$ developed ACLF during hospitalisation. $10(30.3 \%)$ patients had hepatocellular carcinoma. 13 (33.3\%) out of 39 SBP cases had variceal bleeding prior to SBP, with $2(5.1 \%)$ of them occurring during the same admission and the remaining 11 (28.2\%) during prior admission. All patients received prophylactic antibiotics during variceal bleeding as per international guidelines. The demographic data of all subjects is summarised in Table I.

Positive cultures were identified in $33.3 \%$ of the 39 SBP cases. The commonest organism identified was Escherichia coli (46.2\%) followed by Klebsiella spp. (23.1\%) and Enterococcus (23.1\%). ESBL-producing organisms and MDROs were seen in $15.4 \%$ and $5.1 \%$ of the episodes of SBP, respectively. ESBL-producing organisms were more commonly seen in N-SBP compared to C-SBP cases (83.3\% vs. 16.7\%; $p=0.002)$ (Fig. 1). All cases of multidrug resistance occurred in the N-SBP group. Resistance to third-generation cephalosporin and quinolones was observed 
Table I. Baseline demographic data of all patients $(n=33)$.

\begin{tabular}{|c|c|}
\hline Characteristic & No. (\%) \\
\hline Age* $^{*}(y r)$ & $64.5 \pm 10.9$ \\
\hline Male gender & $23(69.7)$ \\
\hline \multicolumn{2}{|l|}{ Aetiology } \\
\hline Hepatitis B & $9(27.3)$ \\
\hline Hepatitis C & $8(24.2)$ \\
\hline Non-alcoholic steatohepatitis & $8(24.2)$ \\
\hline Others & $8(24.2)$ \\
\hline MELD score $^{\dagger}$ & $17.0 \pm 5.6$ \\
\hline MELD-Na score $^{\dagger}$ & $18.0 \pm 6.1$ \\
\hline \multicolumn{2}{|l|}{ Child-Pugh class } \\
\hline A & $0(0)$ \\
\hline B & $20(60.6)$ \\
\hline C & $13(39.4)$ \\
\hline ECOG score ${ }^{\ddagger}$ & $1(0-3)$ \\
\hline \multicolumn{2}{|l|}{ ECOG class } \\
\hline 0 & $15(45.5)$ \\
\hline 1 & $8(24.2)$ \\
\hline 2 & $5(15.2)$ \\
\hline 3 & $5(15.2)$ \\
\hline Hepatic encephalopathy & $5(15.2)$ \\
\hline Previous variceal bleeding & $7(21.2)$ \\
\hline Mean arterial pressure* $(\mathrm{mmHg})$ & $70.0 \pm 10.1$ \\
\hline Heart rate $^{\S}(\mathrm{bpm})$ & $94.8(66-140)$ \\
\hline CLIF-SOFA score* & $7.2 \pm 1.1$ \\
\hline ACLF $^{\sharp}$ & $11(33.3)$ \\
\hline SIRS & $20(60.6)$ \\
\hline Ascitic fluid PMN ${ }^{5}$ (cells $/ \mathrm{mm}^{3}$ ) & $1,418(196-9,010)$ \\
\hline Serum leucocyte ${ }^{\S}\left(\right.$ cells $\left.\times 10^{3} / \mu \mathrm{L}\right)$ & $9.3(2.0-42.0)$ \\
\hline International normalised ratio ${ }^{\S}$ & $1.3(1.0-2.2)$ \\
\hline Bilirubin $^{\S}(\mu \mathrm{mol})$ & $51.0(2.6-196.3)$ \\
\hline Albumin* (g/L) & $27.2 \pm 7.6$ \\
\hline Serum creatinine ${ }^{\S}(\mu \mathrm{mol} / \mathrm{L})$ & $160.4(45.0-677.0)$ \\
\hline Serum sodium* $(\mathrm{mmol} / \mathrm{L})$ & $135.4(5.0)$ \\
\hline Duration from hospitalisation to SBP§ (day) & $0(0-30)$ \\
\hline Antibiotic use in previous $3 \mathrm{mth}$ & $17(51.5)$ \\
\hline Beta-blocker use & $18(54.5)$ \\
\hline PPI use prior to SBP & $24(72.7)$ \\
\hline
\end{tabular}

Data presented as *mean \pm standard deviation, tmedian \pm standard deviation, fmean (range), §median (range). IACLF is defined using European Association for the Study of the Liver criteria based on CLIF-SOFA score. ACLF: acute-onchronic liver failure; CLIF-SOFA: chronic liver failure-sequential organ failure assessment; ECOG: Eastern Cooperative Oncology Group performance status; MELD: Model for End-stage Liver Disease; MELD-Na: Model for End-stage Liver Disease with sodium; PMN: polymorphonuclear leucocytes; PPI: proton-pump inhibitor; SBP: spontaneous bacterial peritonitis; SIRS: systemic inflammatory response syndrome

in $12.8 \%$ and $10.3 \%$ of cases, respectively. The trend of ESBLproducing organisms and MDROs remained low and stable from 2014 to 2017 (Fig. 2). Gram-positive organism was found in 3 (7.7\%) out of 39 SBP cases (two Streptococcus spp. and one Enterococcus spp.).

The prevalence of N-SBP in our cohort was 10 (25.6\%) out of 39 cases. The baseline demographics were similar between

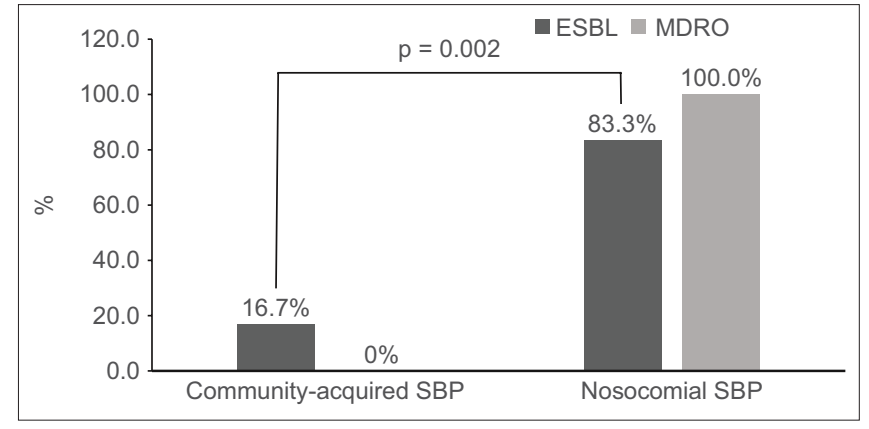

Fig. 1 Bar chart shows the distribution of extended-spectrum betalactamase-producing (ESBL-producing) organisms and multidrug-resistant organisms (MDROs) in community-acquired spontaneous bacterial peritonitis (SBP) and nosocomial SBP. ESBL-producing organisms were more commonly seen in nosocomial SBP compared to community-acquired SBP cases $(p=0.002)$

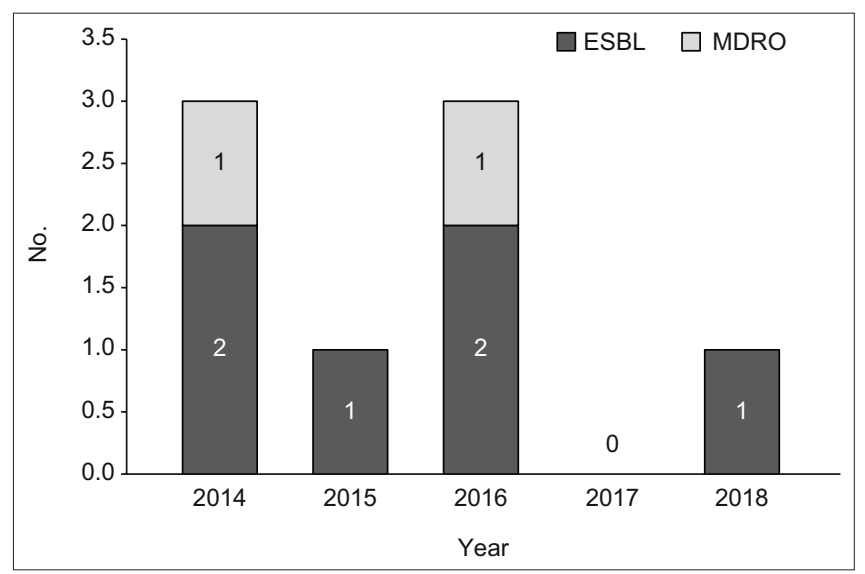

Fig. 2 Bar chart shows the trend of resistant organisms of spontaneous bacterial peritonitis from 2014 to 2017. ESBL: extended-spectrum betalactamase-producing; MDRO: multidrug-resistant organism

patients with C-SBP and N-SBP with respect to age, gender, aetiology of cirrhosis, severity of cirrhosis (Child-Pugh, MELD and MELD-Na scores), cirrhosis-related complications (refractory ascites, previous variceal bleeding and hepatic encephalopathy), proton-pump inhibitor usage and beta-blocker usage. Compared to C-SBP, N-SBP was associated with previous antibiotic use in the past three months $(44.8 \%$ vs. $90.0 \% ; p=0.014)$ and prolonged hospitalisation ( $11.7 \pm 9.2$ days vs. $38.3 \pm 31.6$ days; $p=0.011)$ (Table II).

Overall, 30- and 90-day mortality rates of SBP were 30.8\% and $51.3 \%$, respectively. $\mathrm{N}$-SBP had significantly shorter median survival compared to C-SBP $(2.0 \pm 2.0$ months vs. $5.0 \pm 11.5$ months; $p=0.022$ ). Univariate and multivariate logistic regression analyses of clinical outcomes for SBP are shown in Tables III and IV. Regarding short-term outcomes, a MELD score $>20$ (odds ratio [OR] 13.9, 95\% confidence interval [CI] 2.1-93.7) was predictive of 30-day mortality in SBP. For longterm outcomes, a MELD score $>20$ (OR 3.3, 95\% Cl 1.1-10.2) and the presence of $\mathrm{N}$-SBP (OR 5.2, 95\% Cl 1.2-22.7) were predictive of 90-day mortality in SBP. Non-selective beta-blocker (NSBB) usage was associated with lower 90-day mortality in SBP (OR 0.3, 95\% Cl 0.01-0.97). While culture-positive SBP was associated with a trend of higher mortality, this did not achieve statistical significance. 
Table II. Comparison of demographics between community-acquired and nosocomial cases of SBP $(n=39)$.

\begin{tabular}{|c|c|c|c|}
\hline \multirow[t]{2}{*}{ Variable } & \multicolumn{2}{|c|}{ No. (\%) } & \multirow[t]{2}{*}{ p-value } \\
\hline & C-SBP $(n=29)$ & $N-S B P(n=10)$ & \\
\hline Age $^{*}(y r)$ & $64.4 \pm 10.2$ & $65.9 \pm 11.9$ & 0.669 \\
\hline Male gender & $23(79.3)$ & $6(60.0)$ & 0.213 \\
\hline Aetiology & & & 0.915 \\
\hline Hepatitis B & $7(24.1)$ & $2(20.0)$ & \\
\hline Hepatitis C & $8(27.6)$ & $2(20.0)$ & \\
\hline Non-alcoholic steatohepatitis & $8(27.6)$ & $3(30.0)$ & \\
\hline Others & $6(20.7)$ & $3(30.0)$ & \\
\hline MELD score $^{\dagger}$ & $18(6-34)$ & $18(10-25)$ & 0.912 \\
\hline Child-Pugh class & & & 0.62 \\
\hline B & $17(58.6)$ & $6(60.0)$ & \\
\hline $\mathrm{C}$ & $12(41.4)$ & $4(40.0)$ & \\
\hline Hepatic encephalopathy & $6(20.7)$ & $4(40.0)$ & 0.213 \\
\hline Previous variceal bleeding & $8(27.6)$ & $5(50.0)$ & 0.181 \\
\hline CLIF-SOFA score* & $7.5 \pm 1.3$ & $7.0 \pm 1.2$ & 0.716 \\
\hline ACLF $^{\ddagger}$ & $19(65.5)$ & $7(70.0)$ & 0.56 \\
\hline SIRS & $15(51.7)$ & $7(70.0)$ & 0.265 \\
\hline Antibiotic use in previous $3 \mathrm{mth}$ & $13(44.8)$ & $9(90.0)$ & $0.014^{n}$ \\
\hline Length of stay ${ }^{\S}$ (day) & $11.7 \pm 9.2$ & $38.3 \pm 31.6$ & $0.011^{\pi}$ \\
\hline Duration from hospitalisation to $\mathrm{SBP}^{+}$(day) & $0(0-1)$ & $7(2-30)$ & $<0.001^{9}$ \\
\hline
\end{tabular}

*Data is presented as mean \pm standard deviation, tmedian (range). $¥ A \mathrm{ACLF}$ is defined using European Association for the Study of the Liver criteria based on CLIF-SOFA score. \$Data is presented as median \pm standard deviation. Ip-value $<0.05$ is considered statistically significant. ACLF: acute-on-chronic liver failure; C-SBP: community-acquired spontaneous bacterial peritonitis; CLIF-SOFA: chronic liver failure-sequential organ failure assessment; MELD: Model for End-stage Liver Disease; N-SBP: nosocomial spontaneous bacterial peritonitis; SBP: spontaneous bacterial peritonitis; SIRS: systemic inflammatory response syndrome

Table III. Univariate and multivariate analyses of factors associated with 30-day mortality in patients with spontaneous bacterial peritonitis.

\begin{tabular}{|c|c|c|c|c|}
\hline \multirow[t]{2}{*}{ Variable } & \multicolumn{2}{|c|}{ Univariate analysis } & \multicolumn{2}{|c|}{ Multivariate analysis* } \\
\hline & Unadjusted p-value & OR $(95 \% \mathrm{CI})$ & Adjusted p-value & OR $(95 \% \mathrm{Cl})$ \\
\hline Age $>70 \mathrm{yr}$ & 0.042 & $13.4(0-28.9)$ & 0.584 & $1.4(0.4-4.8)$ \\
\hline $\mathrm{N}-\mathrm{SBP}$ & 0.023 & $8.1(0-18.4)$ & 0.084 & $4.8(0.8-28.9)$ \\
\hline MELD score $>20$ & 0.007 & $3.4(0-8.7)$ & $0.007^{\dagger}$ & $13.9(2.1-93.7)$ \\
\hline ECOG score $>0$ & 0.005 & $2.9(1.7-4.1)$ & 0.084 & $4.8(0.1-28.9)$ \\
\hline ESBL organism & 0.034 & $5.18(0-14.3)$ & 0.222 & $3.1(0.5-19.1)$ \\
\hline VRE coloniser & 0.059 & & & \\
\hline MRSA coloniser & 0.965 & & & \\
\hline PPI usage & 0.400 & & & \\
\hline NSBB usage & 0.487 & & & \\
\hline CLIF-SOFA score $>7$ & 0.209 & & & \\
\hline $\mathrm{HCC}$ & 0.233 & & & \\
\hline $\mathrm{HE}$ & 0.398 & & & \\
\hline Variceal bleeding & 0.339 & & & \\
\hline ACLF & 0.988 & & & \\
\hline SIRS & 0.789 & & & \\
\hline MDRO & 0.385 & & & \\
\hline
\end{tabular}

*Multivariate analysis was performed with adjustment for MELD score $>20, \mathrm{~N}-\mathrm{SBP}$, age $>70 \mathrm{yr}$, ECOG score $>0$ and ESBL organism. tp-value $<0.05$ is considered statistically significant. ACLF: acute-on-chronic liver failure; Cl: confidence interval; CLIF-SOFA: chronic liver failure-sequential organ failure assessment; ECOG: Eastern Cooperative Oncology Group performance status; ESBL: extended-spectrum beta-lactamase; HCC: hepatocellular carcinoma; HE: hepatic encephalopathy; MDRO: multidrug-resistant organism; MELD: Model for End-stage Liver Disease; MRSA: methicillin-resistant Staphylococcus aureus; N-SBP: nosocomial spontaneous bacterial peritonitis; NSBB: non-selective beta-blocker; OR: odds ratio; PPI: proton-pump inhibitor; SIRS: systemic inflammatory response syndrome; VRE: vancomycin-resistant Enterococci 
Table IV. Univariate and multivariate analyses of factors associated with 90-day mortality in patients with spontaneous bacterial peritonitis.

\begin{tabular}{|c|c|c|c|c|}
\hline \multirow[t]{2}{*}{ Variable } & \multicolumn{2}{|c|}{ Univariate analysis } & \multicolumn{2}{|c|}{ Multivariate analysis } \\
\hline & Unadjusted p-value & OR $(95 \% \mathrm{Cl})$ & Adjusted p-value & OR $(95 \% \mathrm{Cl})$ \\
\hline $\mathrm{N}-\mathrm{SBP}$ & 0.031 & $3.3(1.7-14.6)$ & $0.028^{+}$ & $5.2(1.2-22.7)$ \\
\hline MELD score $>20$ & 0.049 & $2.7(0-8.7)$ & $0.038^{\dagger}$ & $3.3(1.1-10.2)$ \\
\hline ECOG score $>0$ & 0.003 & $8.14(3.9-12.4)$ & 0.102 & $0.3(0.1-1.2)$ \\
\hline ESBL organism & 0.079 & $5.2(0-14.3)$ & 0.627 & $1.5(0.3-6.9)$ \\
\hline NSBB usage & 0.039 & $2.9(2.50-13.70)$ & $0.045^{\dagger}$ & $0.3(0.01-0.97)$ \\
\hline $\mathrm{HCC}$ & 0.006 & $1.7(2.2-8.6)$ & 0.204 & $2.1(0.7-6.9)$ \\
\hline Age $>70 \mathrm{yr}$ & 0.136 & & & \\
\hline VRE coloniser & 0.107 & & & \\
\hline MRSA coloniser & 0.155 & & & \\
\hline PPI usage & 0.126 & & & \\
\hline CLIF-SOFA score $>7$ & 0.130 & & & \\
\hline $\mathrm{HE}$ & 0.107 & & & \\
\hline Variceal bleeding & 0.238 & & & \\
\hline ACLF & 0.713 & & & \\
\hline SIRS & 0.996 & & & \\
\hline MDRO & 0.160 & & & \\
\hline
\end{tabular}

*Multivariate analysis was performed with adjustment for MELD score $>20, N-S B P, N S B B, H C C, E S B L$ organism and ECOG score $>0$. $t p$-value $<0.05$ is considered statistically significant. ACLF: acute-on-chronic liver failure; Cl: confidence interval; CLIF-SOFA: chronic liver failure-sequential organ failure assessment; ECOG: Eastern Cooperative Oncology Group performance status; ESBL: extended-spectrum beta-lactamase; HCC: hepatocellular carcinoma; HE: hepatic encephalopathy; MDRO: multidrugresistant organism; MELD: Model for End-stage Liver Disease; MRSA: methicillin-resistant Staphylococcus aureus; N-SBP: nosocomial spontaneous bacterial peritonitis; NSBB: non-selective beta-blocker; OR: odds ratio; PPI: proton-pump inhibitor; SIRS: systemic inflammatory response syndrome; VRE: vancomycin-resistant Enterococci

\section{DISCUSSION}

$\mathrm{N}-\mathrm{SBP}$ is associated with MDROs and poor outcomes. ${ }^{(28-30)}$ The rising trend of multidrug resistance worldwide continues to threaten the sustainability of effective treatments for common infections such as SBP. Because early initiation of appropriate empirical therapy improves survival in N-SBP, and the prevalence of N-SBP varies geographically, ranging from $30.8 \%$ in Korea to $93.3 \%$ in France, such empirical therapy must be guided by knowledge of the local antibiotic resistance profile. . $5,7,25,28,29,31-35)$ In the present study, we reported a relatively lower prevalence (25.6\%) of N-SBP in Singapore. With the low prevalence of resistant organisms among cirrhotic patients with SBP, the current empirical antibiotic recommendations of using ceftriaxone for C-SBP and piperacillin/ tazobactam for N-SBP are reasonable options for Singapore. ${ }^{(36)}$ To our knowledge, no prior study has evaluated the epidemiology and outcome predictors of N-SBP locally. Judicious use of antibiotics is crucial to prevent the rise of ESBL-producing organisms and MDROs in our cohort before we pass the critical point.

We found that N-SBP was strongly associated with ESBLproducing organisms and longer hospitalisation as compared to C-SBP (Table II). These findings were consistent with earlier studies from European and Korean cohorts, in which a high prevalence of resistant organisms was observed. (5,22,37-40) $^{\text {Table } \mathrm{V}}$ shows our review of the existing literature on the outcomes of $\mathrm{N}$-SBP. In our cohort, in contrast to the earlier studies, N-SBP was not associated with higher 30-day mortality (Table III). This may be due to three reasons. First, there was a lower proportion of Child-Pugh Class C cirrhotic patients in our cohort compared to the earlier studies. Second, there was a lower prevalence of ESBL-producing organisms and MDROs in our cohort. Lastly, all our N-SBP patients received adequate empirical coverage (piperacillin/tazobactam). This finding suggests that the administration of appropriate antibiotics following local resistant patterns can improve short-term survival for N-SBP patients.

A MELD score $>20$ and the presence of $\mathrm{N}$-SBP were found to be predictors for 90-day mortality in SBP. A MELD score $>20$ was also found to predict higher 30-day mortality. The MELD score cut-off as a predictor of mortality in SBP was consistent with findings in the published literature. ${ }^{(23,41)}$ This suggests that patients with poorer liver reserve are either more likely to develop SBP or to decompensate following the occurrence of SBP. A MELD score $>20$ could be used as the threshold for initiating more aggressive treatment or closer monitoring among SBP patients. On the other hand, N-SBP was associated with higher 90-day mortality and shorter median survival (Table IV). The high mortality rate among N-SBP cases could be related to gut dysbiosis among patients with advanced cirrhosis. ${ }^{(14)}$ Nevertheless, the question of whether the alteration of gut microbiome observed in N-SBP was the consequence of the disease itself or could potentially lead to more advanced disease remains unanswered.

In our study, the use of NSBBs had a protective effect, reducing 90-day mortality among SBP patients (Table IV). The initial concern about NSBB usage among patients with SBP arose when Mandorfer et al reported a higher risk of hepatorenal syndrome and worse transplant-free survival among 182 SBP patients receiving NSBBs. ${ }^{(42)}$ In contrast, more recent studies showed that NSBBs are associated with the reduction of mortality among patients with SBP in the Danish and CANONIC cohorts. ${ }^{(43,44)}$ These findings suggest that although NSBBs are not 
Table V. Outcomes of studies on nosocomial spontaneous bacterial peritonitis (N-SBP).

\begin{tabular}{|c|c|c|c|c|c|c|}
\hline \multirow[t]{2}{*}{ Author, country, yr } & \multirow[t]{2}{*}{ Study design } & \multicolumn{4}{|c|}{ No. (\%) } & \multirow{2}{*}{$\begin{array}{c}\% \\
\text { 30-day mortality }\end{array}$} \\
\hline & & N-SBP & ESBL-producing organism & MDRO & GPC & \\
\hline $\begin{array}{l}\text { Lutz et al, Germany } \\
(2012-2016)\end{array}$ & $\begin{array}{l}\text { Prospective study, } \\
\mathrm{n}=86\end{array}$ & $58 / 86(68)$ & $27 / 86(31)$ & $0(0)$ & $22 / 86(25)$ & 57.0 \\
\hline $\begin{array}{l}\text { Kim et al, Korea } \\
(2000-2011)\end{array}$ & $\begin{array}{l}\text { Retrospective } \\
\text { study, } n=164\end{array}$ & $123 / 188(65)$ & 9/178 (5) & NA & $43 / 188(23)$ & 41.5 \\
\hline $\begin{array}{l}\text { Cheong et al, Korea } \\
(2000-2007)\end{array}$ & $\begin{array}{l}\text { Retrospective } \\
\text { study, } n=236\end{array}$ & $126 / 236(54)$ & $96 / 236(41)$ & NA & $54 / 236(23)$ & 58.7 \\
\hline $\begin{array}{l}\text { Balaraju et al, India } \\
\text { (2010-2012) }\end{array}$ & $\begin{array}{l}\text { Prospective study, } \\
\mathrm{n}=150\end{array}$ & $20 / 150(13)$ & $72 / 150(48)$ & NA & $2 / 4(50)$ & 62.5 \\
\hline $\begin{array}{l}\text { Campillo et al, France } \\
(1996-2001)\end{array}$ & $\begin{array}{l}\text { Retrospective } \\
\text { study, } n=101\end{array}$ & 94/101 (93) & $12 / 65(19)$ & NA & $32 / 101(32)$ & $49.5^{*}$ \\
\hline $\begin{array}{l}\text { Ariza et al, Spain } \\
(2001-2009)\end{array}$ & $\begin{array}{l}\text { Retrospective } \\
\text { study, } n=200\end{array}$ & $54 / 200(27)$ & $43 / 200(22)$ & NA & $88 / 246(36)$ & 54.2 \\
\hline $\begin{array}{l}\text { Wong et al, Singapore } \\
(2014-2017)\end{array}$ & $\begin{array}{l}\text { Retrospective } \\
\text { study, } n=33\end{array}$ & $10 / 39(26)$ & $6 / 39(15)$ & $2 / 39(5)$ & $3 / 39(8)$ & 40.0 \\
\hline
\end{tabular}

*Inpatient mortality during admission. ESBL: extended-spectrum beta-lactamase; GPC: Gram-positive cocci; MDRO: multidrug-resistant organism; NA: not applicable

harmful in SBP, yet the indiscriminate cessation of NSBB usage among all patients with SBP could be harmful. The decision on NSBB usage should be individualised, and a high dose of NSBB (> $160 \mathrm{mg} /$ day) should be avoided among patients with SBP. ${ }^{(45)}$

The widespread use of quinolone for primary prophylaxis of SBP has been controversial, as it may have contributed to the rising incidence of Gram-positive cocci among SBP cases. Our Gram-positive cocci rate was lower than those in published series. ${ }^{(34)}$ Due to the limitation of measuring ascitic fluid albumin below $20 \mathrm{~g} / \mathrm{L}$, primary prophylaxis of SBP is rarely practised in our institution. Such a practice is likely to be the reason behind an overall low prevalence of Gram-positive organism in our cohort.

We acknowledge that this study had its limitations. First, it had a retrospective and non-randomised design. We tried to address this limitation by adjusting all findings to account for differences in baseline characteristics and potential confounders in multivariate analysis. Second, our results on the demographics of cirrhosis aetiologies and the antimicrobial resistant pattern were derived from a single institution, thus might not be generalisable to another centre. Third, the incidence of SBP may be underdiagnosed, because subjects with clinical suspicion of SBP alone were excluded from this study. Lastly, our sample size is small. A future prospective multicentre study would be ideal to verify our findings.

In conclusion, the prevalence of resistant organisms among $\mathrm{N}$-SBP is low in our population. Early recognition and judicious use of antibiotics can improve survival in N-SBP. A MELD score $>20$ and the presence of N-SBP are predictors of poor outcome among SBP, while the use of NSBBs should be individualised. Our study suggests that the current recommendation for empirical treatment of SBP is appropriate in Singapore.

\section{REFERENCES}

1. Fernández J, Prado V, Trebicka J, et al; European Foundation for the Study o Chronic Liver Failure (EF-Clif). Multidrug-resistant bacterial infections in patients with decompensated cirrhosis and with acute-on-chronic liver failure in Europe. J Hepatol 2019; 70:398-411.
2. Jalan R, Fernandez J, Wiest R, et al. Bacterial infections in cirrhosis: a position statement based on the EASL Special Conference 2013. J Hepatol 2014; 60:131024.

3. Niu B, Kim B, Limketkai BN, et al. Mortality from spontaneous bacterial peritonitis among hospitalized patients in the USA. Dig Dis Sci 2018; 63:1327-33.

4. Piano S, Fasolato S, Salinas F, et al. The empirical antibiotic treatment of nosocomial spontaneous bacterial peritonitis: results of a randomized controlled clinical trial. Hepatology 2016; 63:1299-309.

5. Ariza X, Castellote J, Lora-Tamayo J, et al. Risk factors for resistance to ceftriaxone and its impact on mortality in community, healthcare and nosocomial spontaneous bacterial peritonitis. J Hepatol 2012; 56:825-32

6. Reuken PA, Pletz MW, Baier M, et al. Emergence of spontaneous bacterial peritonitis due to enterococci - risk factors and outcome in a 12-year retrospective study. Aliment Pharmacol Ther 2012; 35:1199-208.

7. Lutz P, Nischalke HD, Strassburg CP, Spengler U. Spontaneous bacteria peritonitis: the clinical challenge of a leaky gut and a cirrhotic liver. World J Hepatol 2015; 7:304-14.

8. Arab JP, Martin-Mateos RM, Shah VH. Gut-liver axis, cirrhosis and portal hypertension: the chicken and the egg. Hepatol Int 2018; 12(Suppl 1):24-33.

9. Such J, Guardiola JV, de Juan I, et al. Ultrastructural characteristics of dista duodenum mucosa in patients with cirrhosis. Eur J Gastroenterol Hepatol 2002; 14:371-6.

10. $\mathrm{Xu} W \mathrm{~W}, \mathrm{Wu} \mathrm{XJ}, \mathrm{Li} J \mathrm{~S}$. Influence of portal pressure change on intestinal permeability in patients with portal hypertension. Hepatobiliary Pancreat Dis Int 2002; 1:510-4.

11. Pascual S, Such J, Esteban A, et al. Intestinal permeability is increased in patients with advanced cirrhosis. Hepatogastroenterology 2003; 50:1482-6.

12. Kalaitzakis E, Johansson JE, Bjarnason I, Björnsson E. Intestinal permeability in cirrhotic patients with and without ascites. Scand J Gastroenterol 2006; 41:326-30.

13. Qin N, Yang F, Li A, et al. Alterations of the human gut microbiome in liver cirrhosis. Nature 2014; 513:59-64

14. Macnaughtan J, Jalan R. Clinical and pathophysiological consequences of alterations in the microbiome in cirrhosis. Am J Gastroenterol 2015; 110:1399410; quiz 1411.

15. Runyon BA. Low-protein-concentration ascitic fluid is predisposed to spontaneous bacterial peritonitis. Gastroenterology 1986; 91:1343-6.

16. Andreu M, Sola R, Sitges-Serra A, et al. Risk factors for spontaneous bacteria peritonitis in cirrhotic patients with ascites. Gastroenterology 1993; 104:1133-8.

17. Llach J, Rimola A, Navasa $M$, et al. Incidence and predictive factors of first episode of spontaneous bacterial peritonitis in cirrhosis with ascites: relevance of ascitic fluid protein concentration. Hepatology 1992; 16:724-7.

18. de Mattos AA, Costabeber AM, Lionço LC, Tovo CV. Multi-resistant bacteria in spontaneous bacterial peritonitis: a new step in management? World J Gastroenterol 2014; 20:14079-86

19. World Health Organization. Global action plan on antimicrobial resistance. Available at: http://www.who.int/antimicrobial-resistance/publications/globalaction-plan/en/. Accessed January 3, 2019.

20. Fiore M, Maraolo AE, Gentile I, et al. Nosocomial spontaneous bacterial peritonitis antibiotic treatment in the era of multi-drug resistance pathogens: a systematic review. World I Gastroenterol 2017; 23:4654-60. 
21. Kim MJ, Song $\mathrm{KH}$, Kim NH, et al. Clinical outcomes of spontaneous bacterial peritonitis due to extended-spectrum beta-lactamase-producing Escherichia coli or Klebsiella pneumoniae: a retrospective cohort study. Hepatol Int 2014; 8:582-7.

22. Oliveira AM, Branco JC, Barosa R, et al. Clinical and microbiological characteristics associated with mortality in spontaneous bacterial peritonitis: a multicenter cohort study. Eur J Gastroenterol Hepatol 2016; 28:1216-22.

23. Iliaz R, Ozpolat T, Baran B et al. Predicting mortality in patients with spontaneous bacterial peritonitis using routine inflammatory and biochemical markers. Eur J Gastroenterol Hepatol 2018; 30:786-91.

24. Sort P, Navasa M, Arroyo V, et al. Effect of intravenous albumin on renal impairment and mortality in patients with cirrhosis and spontaneous bacterial peritonitis. N Engl J Med 1999; 341:403-9.

25. Shi L, Wu D, Wei L, et al. Nosocomial and community-acquired spontaneous bacterial peritonitis in patients with liver cirrhosis in China: comparative microbiology and therapeutic implications. Sci Rep 2017; 7:46025.

26. Moreau R, Jalan R, Gines P, et al; CANONIC Study Investigators of the EASL-CLIF Consortium. Acute-on-chronic liver failure is a distinct syndrome that develops in patients with acute decompensation of cirrhosis. Gastroenterology 2013; 144:1426-37.

27. Sanchez LO, Gustot T. Multidrug-resistant bacterial infection in patients with cirrhosis. A review. Curr Hepatology Rep 2019; 18:28-35.

28. Cheong $\mathrm{HS}$, Kang $\mathrm{Cl}$, Lee JA, et al. Clinical significance and outcome of nosocomial acquisition of spontaneous bacterial peritonitis in patients with liver cirrhosis. Clin Infect Dis 2009; 48:1230-6.

29. Song JY, Jung SJ, Park CW, et al. Prognostic significance of infection acquisition sites in spontaneous bacterial peritonitis: nosocomial versus community acquired. J Korean Med Sci 2006; 21:666-71.

30. Tandon P, Delisle A, Topal JE, Garcia-Tsao G. High prevalence of antibioticresistant bacterial infections among patients with cirrhosis at a US liver center. Clin Gastroenterol Hepatol 2012; 10:1291-8.

31. Li YT, Yu CB, Huang JR, Qin ZJ, Li LJ. Pathogen profile and drug resistance analysis of spontaneous peritonitis in cirrhotic patients. World J Gastroenterol 2015; 21:10409-17.

32. Campillo B, Richardet JP, Kheo T, Dupeyron C. Nosocomial spontaneous bacterial peritonitis and bacteremia in cirrhotic patients: impact of isolate type on prognosis and characteristics of infection. Clin Infect Dis 2002; 35:1-10.

33. Alexopoulou A, Vasilieva L, Agiasotelli D, et al. Extensively drug-resistant bacteria are an independent predictive factor of mortality in 130 patients with spontaneous bacterial peritonitis or spontaneous bacteremia. World J Gastroenterol 2016; 22:4049-56

34. Fiore M, Maraolo AE, Gentile I, et al. Current concepts and future strategies in the antimicrobial therapy of emerging Gram-positive spontaneous bacterial peritonitis. World J Hepatol 2017; 9:1166-75.

35. Özdoğan OC. Empirical antibiotic treatment of nosocomial spontaneous bacterial peritonitis: should we start direct broader-spectrum antibiotics or not? Turk J Gastroenterol 2016; 27:294-5.

36. European Association for the Study of the Liver. EASL Clinical Practice Guidelines for the management of patients with decompensated cirrhosis. J Hepatol 2018; 69:406-60.

37. Umgelter A, Reindl W, Miedaner M, Schmid RM, Huber W. Failure of current antibiotic first-line regimens and mortality in hospitalized patients with spontaneous bacterial peritonitis. Infection 2009; 37:2-8.

38. Song $\mathrm{KH}$, Jeon JH, Park WB, et al. Clinical outcomes of spontaneous bacterial peritonitis due to extended-spectrum beta-lactamase-producing Escherichia coli and Klebsiella species: a retrospective matched case-control study. BMC Infect Dis 2009; 9:41.

39. Kim J, Kang $\mathrm{Cl}$, Gwak GY, et al. Clinical impact of healthcare-associated acquisition in cirrhotic patients with community-onset spontaneous bacterial peritonitis. Korean J Intern Med 2020; 35:215-21.

40. Tsung PC, Ryu SH, Cha IH, et al. Predictive factors that influence the survival rates in liver cirrhosis patients with spontaneous bacterial peritonitis. Clin Mol Hepatol 2013; 19:131-9.

41. Mandorfer M, Bota S, Schwabl P, et al. Nonselective $\beta$ blockers increase risk for hepatorenal syndrome and death in patients with cirrhosis and spontaneous bacterial peritonitis. Gastroenterology 2014; 146:1680-90.e1.

42. Bang UC, Benfield T, Hyldstrup L, Jensen JE, Bendtsen F. Effect of propranolol on survival in patients with decompensated cirrhosis: a nationwide study based Danish patient registers. Liver Int 2016; 36:1304-12.

43. Mookerjee RP, Pavesi M, Thomsen KL, et al; CANONIC Study Investigators of the EASL-CLIF Consortium. Treatment with non-selective beta blockers is associated with reduced severity of systemic inflammation and improved survival of patients with acute-on-chronic liver failure. J Hepatol 2016; 64:574-82.

44. Madsen BS, Nielsen KF, Fialla AD, Krag A. Keep the sick from harm in spontaneous bacterial peritonitis: dose of beta blockers matters. J Hepatol 2016; 64:1455-6. 